\title{
DIEZ PROPUESTAS PARA CUIDARSE Y CUIDAR EN LAS PROFESIONES SOCIALES. HACIA LA CONSTRUCCIÓN DE UNA CULTURA DEL CUIDADO EN LOS PROFESIONALES
}

\author{
Ten proposals to care for oneself and others in the social professions. \\ Towards the construction of a culture of care for professionals
}

Carmina Puig CRUElls ${ }^{1}$

\section{Resumen}

Los profesionales son el recurso más valioso del sistema de servicios sociales y deben de cuidarse para poder cuidar. Cada día se enfrentan con historias de superación, pero también de dolor y de frustración, que los confrontan con ellos mismos.

El artículo quiere contribuir a la generación de consciencia sobre los efectos que produce en los profesionales trabajar con situaciones de riesgo psicosocial y promover la atención y la proactividad en lugar de instalarse en el malestar. De lo contrario, este malestar se traslada al interior de los equipos y también, en última instancia, a las personas atendidas.

Para ello, se fundamentan diez propuestas teórico- prácticas: la interdependencia, la inteligencia colectiva, el doble cuidado, atender la subjetividad, dotarse de una mirada molecular sobre las situaciones, la confianza, el volver a la palabra, la creatividad y el cuidado, investigar para cuidar y cuidarse y, por último, la supervisión social como forma de atención y autocuidado. Todas ellas son las reflexiones que resultan de una práctica sistematizada y del estudio realizado durante el ejercicio de supervisiones con diez equipos y sesenta profesionales del ámbito psicosocial, con el propósito de contribuir a la construcción de una mirada colectiva y así fundar una cultura del cuidado profesional.

Palabras clave: cuidados profesionales, supervisión, profesionales.

\begin{abstract}
Professionals are the most valuable resource of the social service system and they must take care of themselves to be able to care for others. Every day professionals have to deal with stories of overcoming obstacles, but also with stories of pain and frustration, which make them question themselves.

The article aims to generate awareness about the effects on professionals working with psycho-social risk situations and promote attention to these issues and pro-activeness rather than settling in the discomfort zone. If this is not done, this discomfort is transferred to the work teams and, finally, to the people they have to help.

To this end, ten theoretical-practical proposals are presented: interdependence, collective intelligence, dual care, address subjectivity, acquire a molecular perspective of situations, trust, return to words, creativity and care, research to care for others and care for oneself, and finally social supervision as a way to help others and oneself. These reflections are the results of a systematic practice and of a study done during the supervision of ten teams and sixty professionals in the psychosocial field. We will contribute to generate ideas, strategies and set aside resources to propose a care culture in global sense: towards the people we care for, towards teammates and towards oneself.
\end{abstract}

Keywords: professional care, supervision, professional.

Recibido: 04/08/2015

Aceptado: 20/11/2015

Publicado: 03/12/2015

1. Universidad Rovira i Virgili. Facultad de Ciencias jurídicas. CAMPUS CATALUNYA Despacho 20, edificio D1, $2^{\text {a }}$ planta. Av. Cataluña, 3543002 Tarragona. Departamento de Antropología, Filosofía y Trabajo Social. http://dafits.urv.cat. Correo electrónico: carmina.puig@urv.cat 


\section{Introducción}

Los profesionales sociales hablan sobre el cansancio que sienten, especulan sobre el malestar que sienten. Es muy comentado pero a menudo no se traspasa la barrera del comentario sin una verdadera toma de consciencia. «Si lo tenemos que hacer», dicen unos, o «haré lo que pueda» dicen otros, pero en la práctica se considera que tener cuidado de uno mismo está relacionado con mantener una buena salud y hacer ejercicio físico.

Este artículo huye del enfoque fisiológico y es una primera contribución a una nueva perspectiva, una nueva mirada fruto de la experiencia supervisora que pretende generar estrategias concretas para favorecer la salud mental de los profesionales, al mismo tiempo que maximiza su competencia con las personas atendidas. También aborda los problemas y propuestas que se hacen desde una perspectiva psicosocial, fundamentada principalmente en los autores Bleichmar (2009), Dejours (2006), Leal (2003-2006) i Molinier (20132015), orientados desde el psicoanálisis. El tratamiento de los problemas se relega desde una orientación de la fatiga por compasión o por el trauma vicario planteado por Rothschild (2006) en su obra Ayuda para el profesional que ayuda, así como por los planteamientos psicofisiológicos planteados por Figley, Adams \& Boscarino (2008) y Figley (2014).

Son antiguas las discusiones sobre el estrés y sus efectos en las profesiones sociales y como el compromiso y la responsabilidad pueden conducir a cambios en su bienestar psicológico, físico y espiritual. En el ámbito del trabajo social, quizás el autor más tratado y clásico sea Herbert Freudenberger (1974), quien se ha transformado en un referente cardinal para el abordaje del cansancio en el ámbito social. Justamente en Puig (2005) se analizan estos fenómenos y su expresión profesional.

Las propuestas de cuidado se fundamentan en la idea de que los síntomas de agotamiento profesional, propuesto por Freudenberger, son un problema acuciante y de que es posible la ayuda cuando los profesionales expanden su consciencia y practican activamente algunas de las propuestas psicosociales señaladas. Para ello se crean y delimitan diferentes procesos y propuestas prácticas, fundamentadas principalmente en los autores mencionados.

También se ha problematizado sobre los efectos que tiene sobre los profesionales trabajar con personas y situaciones de violencia, vulnerabilidad y pobreza, así como no poner atención a la idea expuesta por Dejours: «se han desatendido los sufrimientos del trabajo y en general el de las relaciones entre subjetividad y trabajo» (Dejours, 2006: 35).

Otro eje que fundamenta el trabajo multidisciplinario e integrador que se presenta es la participación en procesos de supervisión desde finales de 
los años 80 y la trayectoria de investigación realizada sobre este tema. Esta se sitúa en la intersección de diferentes marcos teóricos: trabajo social, teoría sistémica, teoría psicoanalítica, la ética del cuidado que propone Gilligan, (1985) y las capacidades transformativas descritas por Sharmer, (2007) y Kofman, (2007), que integran artes, prácticas y ciencias que ayudan a evolucionar competencias individuales y colectivas desde diversos ejes: uno desde «el ser» (más interno) y el otro «el hacer» (más externo).

Todo ello se realiza a través de la sistematización de la práctica de las sesiones de supervisión. Esta es entendida como la reconstrucción teórica de una experiencia práctica concreta y realizada (Gagneten, 1987; Aguayo, 1992; Ayllón, 1995). Precisamente a través de la sistematización práctica de las sesiones y de los contenidos de las supervisiones se ha analizado la experiencia como supervisora y de los supervisados, y se han elaborado las diferentes propuestas que se desarrollan en este artículo. Este proceso se ha desarrollado durante los años 2013 y 2014 dialogando con más de diez equipos conformados por sesenta profesionales.

Por último, se exponen las reflexiones que han acontecido como resultado de esta sistematización de supervisiones con profesionales del ámbito psicosocial. Ellos y ellas, sus aportaciones, han guiado las directrices de las propuestas que se formulan, que no tienen otro objetivo que el de amplificar la toma de conciencia sobre las necesidades profesionales y especialmente de los efectos que produce trabajar con situaciones de crisis social y así generar, dentro de lo posible, nuevas prácticas e instrumentos para estar atentos hacia los otros y hacia uno mismo con el objetivo de evitar y prevenir instalarse en el malestar personal, en el malestar en los encuentros con el otro o en el interior de los equipos.

Con ello se intenta favorecer la construcción de una mirada más colectiva y así generar ideas, estrategias y destinar recursos a fundar una cultura del cuidado en sentido global: hacia las personas atendidas, hacia los compañeros y hacia uno mismo.

A continuación se desarrollaran diez propuestas para cuidarse y cuidar en las profesiones sociales. A partir de una instancia de reflexión individual como supervisora y otra colectiva por parte de los supervisados, se ordenará lo acontecido en la experiencia generando conocimientos.

\section{Diez propuestas para cuidarse y cuidar en las profesiones sociales}

Las propuestas se fundamentan en tres ejes: el derecho a cuidar, cuidarte y que te cuiden. Partiendo de la inferencia de que los profesionales son el recurso más valioso del sistema de servicios sociales, educativos y sanitarios, y de que 
se enfrentan cada día a historias de superación, pero también de dolor y de frustración, que son duras de soportar y que los confrontan con ellos mismos.

La primera propuesta se plantea bajo el epígrafe de la interdependencia. Las personas somos interdependientes, nos necesitamos los unos a los otros para sobrevivir. Continuamente estamos intercambiando e interactuando y es precisamente en este intercambio donde surgen los efectos más brillantes y los más miserables de las relaciones. Todos formamos parte de este entramado de dependencias puesto que todos ayudamos.

La ética del cuidado nacida en Estados Unidos, de la mano de Carol Gilligan, reconoce la vulnerabilidad como una condición común de la humanidad. En su obra destaca que somos seres vulnerable extraordinariamente frágiles y dependientes a lo largo de las diversas etapas de la vida y que es necesario aceptar que somos vulnerables, que nos necesitamos los unos a los otros (Gilligan, 1985). Estas afirmaciones se oponen al valor neoliberal de la autonomía total y la independencia de la necesidad del otro. Guilligan afirma que somos interdependientes.

Con los planteamientos neoliberales imperantes y las políticas públicas menguando, las garantías del estado del bienestar están en peligro. En contrapartida el uso de métodos de gestión del mundo industrial, basados en los retos de la competencia económica, están más presentes que nunca en los sectores públicos a través de la gestión del caso en servicios sociales, la gestión sanitaria y también la gestión universitaria. Todos están regidos por las reglas de las buena gestión que si bien son imprescindibles para la administración de los recursos no toma en cuenta el trabajo real de tener cuidado de las personas. Esta situación hace que se tenga que retomar y poner en valor la cultura del cuidado, en el sentido de preocuparse por la atención, por el trato de los otros. No es suficiente con tratar bien técnicamente. Las personas «quieren estar informadas, sentirse seguras, que se ocupen de sus problemas y sus preocupaciones» (Molinier, 2015:2). Esto es más necesario que nunca. Todos lo necesitamos para estar-bien, para dar y darnos bien-estar.

El bienestar atraviesa a los profesionales que trabajan juntos, a los equipos de diferentes instituciones que trabajan desconectados y con poca comunicación. Intercambiar siempre ha sido importante. Ahora acontece como imprescindible porque los espacios de intercambio de atención, de conocimiento consciente y compartido, transforman nuestra manera de vivir y son saludables, curan y hacen bien.

Una segunda premisa es la inteligencia colectiva. Ni todo depende de mí, ni todo depende de los otros. Los acontecimientos y el insuficiente desarrollo del estado del bienestar se han ido tragando una gran cantidad de inteligencia 
colectiva de los profesionales, que ahora se encuentra desperdigada y no encuentra caminos para volver a re-pensar las situaciones. «La sensación de derrota es tan grande que ha dejado a los profesionales, a los intelectuales rendidos no sólo políticamente, sino a nivel de pensamiento. Es como si no se pudieran pensar nuevas opciones. Se ha impuesto una realidad, no la realidad» (Bleichmar, 2009:30).

Ni todo depende de mí ni todo depende de los otros, así es. Esta actitud se refiere a las iniciativas y a las nuevas formas que pueden ejercerse sobre uno mismo y el entorno para que sea más equilibrado. La idea es que no todo viene dado por las condiciones externas. Poder cuidar bien a los demás empieza por uno mismo y por el entorno inmediato. Esto requiere poder preguntarse ¿con quién? ¿Dónde? ¿De qué manera y de qué forma quieres y quieren ser cuidados? ¿Cómo establecer relaciones, debates, construcción conjunta de ideas? ¿En qué tiempos quieres y puedes hacerlo? No todo depende del otro.

La ética del cuidado nos aporta la necesidad de mantener un doble cuidado, hacia nosotros y hacia las personas atendidas. Profesionalmente se está abocado a trabajar muy atentamente en un doble cuidado: por un lado se supone a los profesionales una experiencia y un saber que actúa como resorte de confianza en las personas atendidas, como facilitadores de acciones sociales en beneficio de las personas. Pero cómo escribe Tronto (2013), la atención se estructura como una continuidad con lo que cada uno necesita y nunca como una dicotomía entre los que son atendidos y los cuidadores. Los profesionales necesitan poder reconocer su vulnerabilidad e interdependencia y así quizás poder pensar de nuevo sobre su rol y responsabilidad. «El cuidado no es una moral de los buenos sentimientos, es un proyecto social» (Tronto, 2013:44).

Mantener una atención hacia uno mismo es completamente necesario para no causar daños, para atender a lo que se está sintiendo y no traspasarlo (dentro de lo posible) a las personas atendidas (mal humor, frustración). Igualmente es necesario para controlar las expectativas profesionales y deshacerse de la idea de que trabajar con los otros, o posibilitar el acceso a los servicios, satisface plenamente el espacio personal-profesional. A menudo se exige a los profesionales tomar distancia y separar de forma artificial la vida del trabajo, pero este mandato es contraproducente porque niega la realidad y el riesgo. Los profesionales, siempre subjetivos en tanto que sujetos, sufren, sienten, tienen esperanzas e ilusiones. Es desde el sujeto encarnado en el profesional que se crea empatía y se puede atender al otro. La conciencia del riesgo y de los límites es lo que permite escuchar y aproximarse al otro, y comporta la necesidad de pensar y generar recursos protectores. 
Las transformaciones sociales son tan rápidas que requieren atender a la subjetividad, de lo contrario no es posible asumirlas. No se pueden subjetivar, ni hacerlas nuestras tan deprisa (Leon y Zemelman, 1997). Se quieren superar los problemas y resolver de forma muy rápida. Se quiere todo para ahora mismo.

A menudo la respuesta profesional es actuar, hacer, obteniendo (en parte) gran satisfacción del trabajo realizado, a pesar de saber que cuidar de las personas no es tan solo actuar, dar prestaciones, hacer informes o gestionar.

Para atender a la subjetividad hay que recuperar la espera, que no es la pasividad. Sostener la espera implica que se moviliza un proceso psíquico de reflexión y anticipación en el profesional, que puede resultar útil para elaborar la propia irritación que se produce cuando se trata de no hacer, de no ofrecer un recurso o de respetar otro ritmo profesional (Cancrini, 1991). Atender cuidadosamente nuestra subjetividad es no danzar al ritmo de lo urgente, de lo que es noticia. Estar informado no es conformarse con reunir mucha información sobre las personas. Sabemos que estar enterados, tener sobre o infrainformación, a veces, puede disparar intervenciones y decisiones sin tomar la suficiente distancia que permita que fluya la reflexividad y la calma.

El contexto individualista, basado en la búsqueda de la satisfacción de uno mismo, y que a menudo evita el encuentro con el otro, pudo hacer creer en un estado del bienestar que suplía la propia responsabilidad. Quizás en el imaginario social no nos hacía falta el otro y hoy es posible descubrir que las posiciones del cuidador y de la persona cuidada no son fijas y ambos tienen algo que ofrecerse. Se basan en la relación y no son reducibles a una actividad ni a una actitud de una sola persona sino que se apoya en el trabajo en común.

Se necesita volver a lo colectivo, a creer y sentir que la vida no sólo depende de uno mismo, sino de todos los que nos rodean. El trabajo, el desarrollo profesional, la familia, los amigos, las personas con las que poder actuar. Dependemos de lo colectivo, de aquello que es común, de saber poner en valor nuestro potencial colectivo. Una alternativa seria realizar un trabajo basado en la cooperación, en la atención compartida a las pequeñas cosas.

Dotarse de una mirada molecular es una propuesta proactiva. La atención social está necesitada de una mirada más molecular, más atenta a las transformaciones mínimas que acontecen en los procesos subjetivos de las personas. Precisa concentrarse, detenerse y apoyar la intervención en los procesos minúsculos que acontecen a las personas, también en los momentos de crisis. En términos de cuidado hay que prestar mucha atención a las relaciones, a las conexiones, y a las redes entre personas. 
Por otra parte, se pide más calidad en los servicios y de manera simultánea aumenta la lógica de la gestión y de la evaluación a partir de criterios objetivables. Sin duda es natural que tratemos de tener buena atención y cuidado a la vez que gestionamos de manera óptima los recursos de los ciudadanos. Pero la objetividad evaluadora no siempre es compatible con la realidad del trabajo de cuidar.

La cultura del cuidado implica habilidades discretas, encaje emocional y atención a las pequeñas cosas inefables. Comporta una conversación, entrelazada en la cotidianidad o en la textura de la vida cotidiana, como nos remarca Oury (2007). A veces resulta difícil evaluar este trabajo que a menudo sólo tiene sentido a largo plazo o de manera inesperada. El tiempo de la gestión no siempre coincide con el tiempo del cuidado, más flexible y discontinuo.

La sexta propuesta se fundamenta en la confianza hacia las personas que atendemos y hacia nosotros mismos. La confianza es un factor básico en las personas, tanto para la autoestima como para la convivencia. Se necesita recuperar la confianza en nuestros representantes, pero también la confianza en los compañeros, en las familias, en los jóvenes con los que trabajamos. La desconfianza es un tóxico que deteriora y debilita.

Poder recuperar la confianza en las personas está en relación con la espera atenta del otro, sostenida por el profesional. Consiste en realidad en construir un espacio de umbral (González, 2013) ${ }^{2}$, normalmente entre dos puertas, pero simbólicamente un espacio de tránsito donde las personas atendidas no están ni integradas (dentro del sistema) ni excluidas (fuera del servicio). Están en un espacio evolutivo intermedio, de difícil evaluación debido a su invisibilidad, pero que les ayuda a construir su confianza, su capacidad de superación, y sus acciones o reacciones a lo que les está sucediendo. Es un espacio de valor incalculable pero que no se puede medir bajo las leyes de la economía de mercado.

Ahora bien, también hace falta confianza en uno mismo como profesional para poder decir no, poder decir basta, para poder poner límites. Cuidarse implica esfuerzo, conocimientos, preguntarse cómo se conoce y se trata al otro, cooperando y reflexionando conjuntamente. Son precisamente este conjunto de prácticas de cuidado las que están llenas de decisiones donde no hay reglas fijas sino contextuales, que implican reflexión y tiempo improductivo (Molinier, 2013).

2. Conferencia pronunciada 12/3/2013 por Itziar González. Arquitecta, con motivo de la entrega de la medalla de oro del Colegio profesional del Trabajo Social. Catalunya. Recuperado el 1 de junio de 2015 https://youtu.be/xV1IHuRVSAk. 
Para todo ello hay que poder hacerse confiable. Ser más claro y riguroso, si cabe, en las narraciones sobre la realidad que se trabaja. Explicarse mejor en los entornos de influencia; exponer con transparencia los marcos desde donde se puede trabajar y manifestar posicionamientos. Todo ello otorga mayor credibilidad a los sistemas desde donde se actúa y hace más confiables a los profesionales.

Abandonar lo que Bleichmar (2009) denomina como una autocensura, que no sólo está condicionada por ser funcionario o pertenecer a una determinada empresa, sino por algo menos tangible, que tiene que ver con la falta de una perspectiva compartida respecto a la posibilidad de esbozar o diseñar algún tipo de propuesta nueva. Ella lo atribuye al malestar sobrante, en el sentido de que este malestar viene dado por la falta de un proyecto, por el sometimiento a la idea de que las cosas son así y siempre serán así, y que lo único que podemos hacer es amoldarnos a lo que hay (Bleichmar, 2009). De este modo, como dice Muraro (2012), nos reducimos a una existencia disminuida.

Es necesario recuperar el pensamiento de las cosas, sobre lo que pasa y sucede en el entorno, más allá de la inmediatez, sin asumir paradigmas insostenibles de atención que alejan del conocimiento de la realidad y colocan en una repetición ritualitzada (PIRMIS, ayudas económicas...) que abocan al desaliento y a la parálisis intelectual.

Quizás nos sobran imágenes, actos. Para ello hay que volver a la palabra. Faltan palabras, conversaciones, diálogos para generar alternativas o soluciones. Se necesita volver a la palabra, nombrar lo que pasa desde uno mismo y no desde la agenda de los otros (políticos, responsables de servicios...).

También la priorización durante las últimas décadas de un trabajo individualizado, masivo e invasivo, ha generado pérdida de palabras, experiencias y saber que otras generaciones profesionales tenían sobre el trabajo con grupos y colectividades.

El regreso a la palabra abre nuevas posibilidades, ya que «el lenguaje es generador, da orden y sentido a nuestra vida y a nuestro mundo y opera como una forma de participación social» (Anderson, 2000:22). El regreso a la palabra significa también recuperar el diálogo intergeneracional, el diálogo intercultural, y sobre todo el diálogo entre profesionales. Tan solo el diálogo, la palabra y el trabajo colaborativo puede conducir a una mayor creatividad. Se necesita creatividad para generar alternativas de trabajo, de acción social, alternativas que tienen que recuperar aquello que sabíamos hacer cuando existían menos recursos económicos. La innovación social necesita de la 
capacidad para impulsar prácticas concretas y sostener la impotencia, y así generar alternativas. ${ }^{3}$

La conciliación entre la creatividad y el cuidado profesional son imprescindibles. La creatividad es necesaria para reconocer y participar en las iniciativas sociales que acontecen en territorios concretos o en los servicios y requiere interés para saber lo que saben los otros, las personas que se atienden, los compañeros de profesión u otros servicios del territorio. Hay que resaltar la importancia de incorporar la perspectiva del usuario en los modelos de intervención y evitar dentro de lo posible las rutinas cotidianas acríticas.

Cuidar conlleva disponer de creatividad técnica para dotar a los recursos y las prestaciones de un nuevo significado. Los recursos no pueden cubrir de manera total lo que las personas esperan de los servicios. Los profesionales pueden acompañar procesos, mostrar posibilidades o distribuir prestaciones, pero el grado de satisfacción de las personas atendidas y el de los profesionales es limitado. Es recomendable no caer en un sobre exceso de valoración del poder transformador del recursos (Puig, 2008a).

Se necesita re- significar los recursos, en el sentido de dotarlos de nuevos significados. Estos se deben utilizar en su justa medida porque en caso contrario se devalúa su valor y el poder de cambio que tiene un recurso bien indicado y orientado (Puig, 2008b).

Es importante prestar atención al modelo de gestión imperante: primero el recurso y después la persona. A menudo los profesionales son presionados para resolver rápidamente los problemas o las listas de espera, cuando todo ello es muy complejo, y se sabe que algunos problemas solo pueden desaparecer a largo plazo y algunos no tienen solución. No creer en la validez del recurso significa descuidarse profesionalmente, ya que como indica Leal (2006),» lo que genera más incomodidad en el profesional no es la necesidad que le expresa el usuario sino la posición que éste adopta ante la misma. Quizás la necesidad sea objetivable [...] pero el sujeto necesitado, el que expresa yo necesito... me hace falta..., apela a la subjetividad y apunta a la subjetividad del que escucha» (Leal, 2006:37). Hay que avanzar hacia una re-significación de los recursos materiales. Con este propósito, planteé en algunos artículos de 2008 diferentes maneras de aproximación a las prestaciones y a los recursos, y a las que denominé micro-intervenciones para la mediación de los recursos, y que ahora denominaría prácticas de cuidado. Estas se refieren a orientaciones que sitúan al recurso en su capacidad real de apoyo. Respetando los

3. Enrique Sacanell (2009). Curso sobre calidad servicios sociales. Recuperado el 27 de mayo 2015 http://es.slideshare.net/sacanell/curso-calidad-servicios-sociales-2009-eudel-ivap. 
requisitos de una prestación sin usarla como reparación de lo que no existe en el sistema. De lo contrario, como expone Leal, las personas no pueden aceptar las prestaciones como funciones neutrales y es el vínculo y la implicación lo que fatiga y agota a los profesionales. Es necesario reconocer que aunque las instituciones han hecho un esfuerzo para dotarse de protocolos estandarizados, con ellos se afrontan las necesidades pero no los efectos vinculados los mismos.

Investigar para cuidar y cuidarse. La investigación es una manera de autocuidado. Esta se puede hacer investigando sobre la práctica y la acción profesional a través de la sistematización. Son necesarias investigaciones que aporten construcciones teóricas desde la experiencia práctica concreta. Se trataría de partir de la experiencia profesional como unidad sometida a un proceso de análisis (Aguayo, 1992).

Pero ¿cómo cuida la investigación a los profesionales? La investigación nos da conocimiento sobre aquello que se ha hecho y ha resultado útil y ha generado bienestar en las personas y ayuda a identificar aquellas actuaciones profesionales sin sentido y que no se tendrían que repetir. Permite identificar formas alternativas de hacer acción social que superen las estructuras rígidas de algunos encargos institucionales. Para realizar investigación y docencia debemos que proponer marcos, pedir tiempo reconocido y recursos en nuestras instituciones y así poder repensarse como profesionales y hacerlo colectivamente.

La supervisión social es una forma de atención y autocuidado. La supervisión es por excelencia una forma de atención, de cuidado de los profesionales ${ }^{4}$. La supervisión, como espacio de renovación durante el ejercicio profesional, se caracteriza por ser un ámbito de reflexión sistemática sobre la acción profesional sin cumplir funciones de control. Es un espacio para preguntar, para exponer dudas, para canalizar la no solución y debatir conflictos. El grupo y el supervisor escuchan y permiten que fluya la subjetividad de los participantes (Puig, 2014; Hernández, 2000).

La supervisión se basa en la experiencia y la práctica profesional, con el fin de mejorarla. Su función es pensar sobre lo que se hace, sobre el trabajo, para devolverlo a las personas atendidas. Este es su gran potencial formativo. Permite que puedan ser reconocidos problemas y experiencias dolorosas que se sufren en el trabajo, y tiene efectos terapéuticos en la medida que contiene, trata y posibilita, la elaboración de dificultades de forma cooperativa. El

4. Entrevista Carmina Puig. «Los profesionales somos el recurso más valioso del sistema de atención a las personas y debemos cuidarnos para cuidar» Obtenida 4 de junio 2015. http://www.social.cat/entrevistes 
supervisor no tiene funciones de control, ni de coordinación, ni de dirección. Por este motivo es una condición indispensable que el supervisor sea independiente y foráneo a la institución contratante. Es justamente esta posición del supervisor, distante de la tarea y de la institución, la que se convierte en una oportunidad para el supervisado de descubrir fortalezas personales y profesionales.

En las sesiones de supervisión se pueden tratar casos o los efectos de las situaciones institucionales en la intervención profesional. Aquello que permite intervenir al supervisor es el acuerdo de los participantes de mantener un espacio en el cual estén garantizadas la escucha, la palabra, la participación y la confidencialidad. Para los equipos, la supervisión resulta muy conveniente y aconsejable, a veces imprescindible y siempre útil (Puig, 2009).

En Cataluña diferentes instituciones han hecho suya esta práctica. Concretamente, existen experiencias de convenios entre colegios profesionales y diputaciones, o entre universidades y administraciones locales o comarcales, que han contratado servicios de supervisión externa y de este modo han apoyado a sus profesionales. ${ }^{5}$

La supervisión es una herramienta que, después de décadas de prácticas, es muy útil en la lectura de la complejidad actual (Puig, 2011:23) y en la construcción de una cultura de autocuidado profesional.

\section{Referencias bibliográficas}

Aguayo, C. (1992). Fundamentos teóricos de la sistematización. Revista de Trabajo Social de la Universidad Católica de Chile, 61, 31-16.

ANDERSON, H. (2000). Conversación, lenguaje y posibilidades: Un enfoque posmoderno de la terapia. Buenos Aires: Amorrortu.

AYLlón, M. R. (1995). La sistematización de la práctica. Apuntes desde una experiencia docente. Revista de Trabajo Social de la Universidad Católica de Chile, $61,2-28$.

Bleichmar, S. (2009). Superar la inmediatez: Un modo de pensar nuestro tiempo. Buenos Aires: Ediciones del CCC (Centro Cultural de la Cooperativa F. Gorini).

Dejours, C. (2006). La banalización de la injusticia social. Buenos Aires: Topia editorial.

Gilligan, C. (1985). In a Different Voice: Psychological Theory and Women's Development. Cambridge, Massachussets: Harvard University Press, 1982.

5. Col.legi Oficial de Treball Social de Cataluña. Obtenido el 4 junio 2015. http://www. tscat.cat/ 
Traducción: La moral y la teoría. Psicología del desarrollo femenino. México: Fondo de Cultura Económica.

CANCRINI, L. (1991). La psicoterapia, gramática y sintaxis. Barcelona: Paidós.

Gagneten, M. (1987). Hacia una metodología de sistematización de la práctica. Buenos Aires: Humanitas.

HERNANDEZ, J. (2000). La supervisión. Un sistema de asesoramiento y orientación para la formación y el trabajo. Valencia: Nau LLibres.

Kofman, F. (2007). Metamanagement. LHospitalet del Llobregat: Granica.

LEAL. J. (2003). Motivació i desencant profesional. Fórum. Revista del Centre d'Estudis Jurídics i Formació especialitzada. 1. Generalitat de Catalunya.

LEAL, J. (2006). La continuidad de cuidados y trabajo en red en salud mental. Madrid: Asociación Española de Neuropsiquiatría.

LeÓn, E., Zemelman, H. (1997). Subjetividad: umbrales del pensamiento social. México: Ed. Anthropos.

Molinier, P. (2013). Le Travail du care. París: La disopute / Snédit.

Molinier, P. (2015). Salud y trabajo en trabajadores hospitalarios: cultura de la gestión, cultura del cuidado, ¿Una condición imposible? Revista Topia. Recuperado de http://www.topia.com.ar/articulos/salud-y-trabajotrabajadores-hospitalarios-cultura-gestioncultura-del-cuidado-\%C2\%BFuna

Muraro, L. (2012). El poder y la política no son lo mismo. Revista Duoda, 39. Universidad de Barcelona, 37, 47-59.

OuRY, J. (2007). Psychothérapie institutionnelle, histoire et actualité. Paris: Éditions du Champ Social.

PUIG, C. (2005). El agotamiento de los profesionales. La importancia de la supervisión. Revista Trabajo social y salud, 50, 11-25.

PuIG, C. (2008a). La intervención social: más allá del recurso más cerca del vínculo. Servicios sociales y política social, 82, 9-27.

PUIG, C. (2008b). Las posiciones institucionales y profesionales que se imbrican en la atención a la demanda de las personas en los servicios sociales: la mediación entre la demanda y los recursos ofertados. Documentos Trabajo social, 40, 195-208.

PUIG, C. (2009). El equipo, las coordinación y la supervisión: construyendo la red. Sistemas de trabajo que contribuyen al trabajo en red. Aportación singular de la supervisión. VII Jornadas de servicios sociales de Atención Primaria. Colegio de educadoras y educadores sociales de Cataluña, 157-168.

PUIG, C. (2011). La supervisión en los equipos de Servicios Sociales: una oportunidad para la reflexión, el pensamiento y el cuidado de los profesionales. Cuadernos de Trabajo Social, 24, 123-133. Universidad Complutense de Madrid. 
PUIG, C. (2014). Supervision of child social care teams: a method to ensure quality services. Revista de cercetare [i interven] ie social 4 . The online version: www.scopus.com

SHARMER, O. (2007). Theory U: Leadingfrom the future as it Emerges. Cambridge. MA: Society for organizational Learning.

Tronto, J.C. (2013). Caring Democracy. Markets, equality and justice. New York: University Press. 